\title{
Atlas-Driven Scan Planning for High-Resolution Micro-SPECT Data Acquisition based on Multi-View Photographs: a Pilot Study
}

\author{
Martin Baiker ${ }^{\mathrm{a}}$, Brendan Vastenhouw ${ }^{\mathrm{b}, \mathrm{d}}$, Woutjan Branderhorst ${ }^{\mathrm{b}}$, Johan H.C. Reiber ${ }^{\mathrm{a}}$, \\ Freek Beekman ${ }^{\mathrm{b}, \mathrm{c}, \mathrm{d}}$, Boudewijn P.F. Lelieveldt ${ }^{\mathrm{a}, \mathrm{c}}$ \\ ${ }^{a}$ Div. of Image Processing, Leiden University Medical Center, Leiden, The Netherlands \\ ${ }^{b}$ Image Sciences Institute, University Medical Center Utrecht, The Netherlands \\ ${ }^{\mathrm{c}}$ Delft University of Technology, Delft, The Netherlands \\ ${ }^{\mathrm{d}}$ MILabs BV, Utrecht, The Netherlands
}

\begin{abstract}
Highly focused Micro-SPECT scanners enable the acquisition of functional small animal data with very high-resolution. To acquire a maximum of emitted photons from a specific structure of interest and at the same time minimize the required acquisition time, typically only a small subvolume of the animal is scanned that contains the organs of interest. This Volume of Interest (VOI) can be defined manually based on photographs of the animal taken prior to SPECT scanning, for example two lateral views and a top view. In these photographs however, only the surface of the animal is visible and therefore visual estimation of the location of these organs may be difficult.

In this paper, we propose a novel atlas-based technique for estimating the organ VOI for the major organs by mapping a small animal atlas to optical scout images. The user is required to outline the animal contour in one lateral view, and to mark two lateral landmarks in the top view photograph. These landmarks subsequently serve as fiducial landmarks to define a 3D Thin-Plate-Spline mapping of an anatomical mouse atlas to the photographic coordinate space. Planar projections of the mapped atlas organs on the photographs greatly facilitate the estimation of the size and position of the target organ. To validate the proposed approach, the estimated organ VOIs were compared to manually drawn organ outlines in a Micro-CT scan, which was co-registered to the scout photographs using physical landmarks. The results demonstrate a highly promising volume correspondence between the real and the estimated organ VOIs.
\end{abstract}

Keywords: small animal Micro-SPECT, scan planning, Volume of Interest definition, atlas mapping, thin-plate spline

\section{INTRODUCTION}

Image acquisition using clinical imaging modalities such as Magnetic Resonance Imaging (MRI) or Single Photon Emission Computed Tomography (SPECT), is usually performed by a specialist that indicates a particular scanning VOI. Depending on the target it is necessary to define the location, the size and eventually the orientation of the VOI. To facilitate this, usually a scout is generated. For MRI imaging, this means that a larger part of the subject is scanned with low resolution first. Subsequently this scout can be used to refine the scan VOI which greatly reduces the acquisition time required for the high resolution scan. Using SPECT imaging, this procedure may be applied as well as long as the concentration of the radioactive tracer is still sufficiently high after the scout scan. If this cannot be assured or if the dynamic uptake of the tracer rather than its presence is of interest, image data of a second, co-registered modality might be utilized for scan planning. Besides the reduced acquisition time, tight scan VOI selection has an additional advantage when using pinhole imaging like pinhole SPECT, since there is a strong correlation between the scan VOI and the obtainable data resolution [1].

In practice, even with a scout available, defining the VOI for a specific target may be a difficult task, even for an experienced scanner operator. Above that, the procedure is time consuming, subject to inter- and intra-person variability and difficult to reproduce. This is especially critical for follow-up studies where the same subject has to be scanned multiple times at subsequent points in time, because to facilitate a comparison of the data it should be acquired with the same protocol and at the same resolution.

Medical Imaging 2009: Visualization, Image-Guided Procedures, and Modeling,

edited by Michael I. Miga, Kenneth H. Wong, Proc. of SPIE Vol. 7261, 72611L

(C) 2009 SPIE · CCC code: 1605-7422/09/\$18 · doi: 10.1117/12.813516

Proc. of SPIE Vol. $726172611 \mathrm{~L}-1$ 
In this work we present a method to assist a scanner operator in defining the scan VOI for small animal Micro-SPECT data acquisition. It is based on the U-SPECT-II system from MILabs [2] (MILabs B.V., Utrecht, The Netherlands), which is the first nuclear scanner with a $3 \mathrm{D}$ method to focus pinholes. In addition it is supplied with optical hardware and a dedicated interface to roughly locate structures of interest. A schematic overview of the scanner is given in Figure 1 (A). Currently, a subject is put onto a motorized animal holder first and subsequently, scan planning is performed by means of three photographs (scout views), which are taken from three different orthogonal views: a top view and two lateral views. Then the scanner operator needs to assign a rectangular box in two of the views (red box in Figure 1, B) such, that a particular organ of interest is fully enclosed by the selection. Since the optical cameras are calibrated to the U-SPECT reference, the thus defined three dimensional VOI can directly be used to guide the data acquisition. With the current setting, the VOI definition procedure is complicated by the fact that only the animal surface is visible and therefore tight localization of a particular internal structure is very difficult and time consuming.

The contribution of this work is twofold:

- To introduce a method to facilitate the scan VOI definition by using a-priori knowledge of internal anatomical structures. To this end an anatomical animal atlas is used that is individualized based on a sparse set of optical photographs.

- To evaluate the accuracy of the automated VOI definition by comparing it with the true anatomy derived from a co-registered Micro-CT dataset. Therefore, 3D dice indices of volume overlap are calculated for major organs: brain, heart, kidneys and the lungs and scores are determined that indicate if an automatically determined VOI does entirely enclose the target organ.

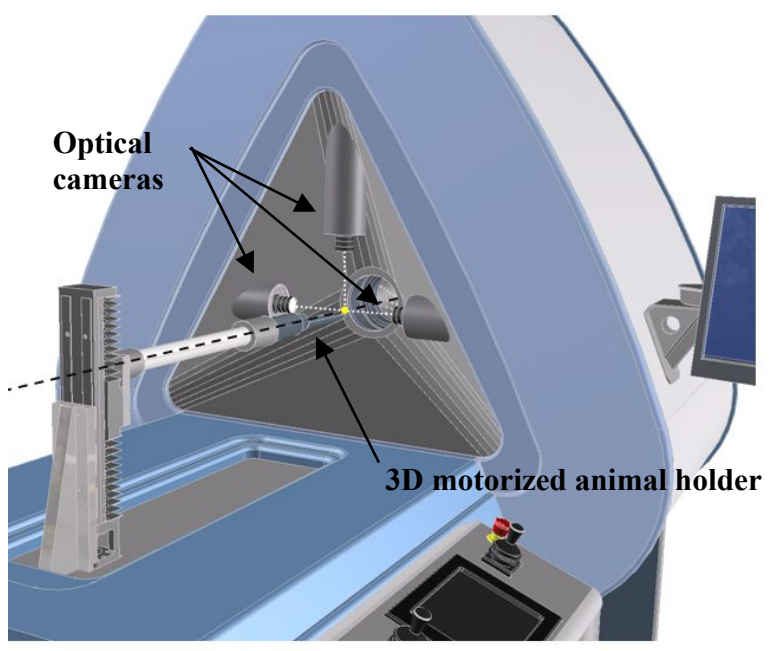

(A)

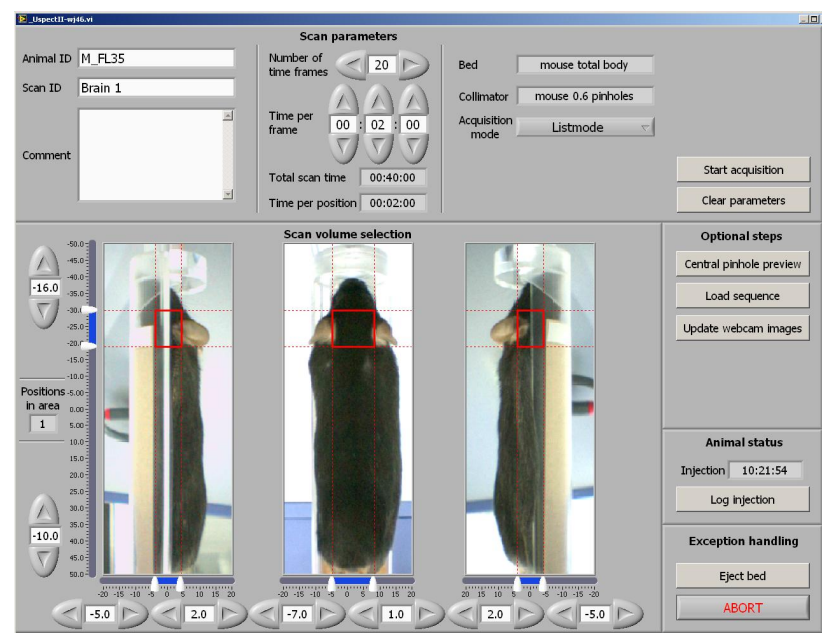

(B)

Figure 1: Equipment to perform scan planning and U-SPECT data acquisition. (A) Schematic of the Micro-SPECT scanner U-SPECT-II. (B) The graphical user interface for scan planning with the U-SPECT-II showing three photographs of a mouse, taken with the optical cameras indicated in (A). The scanner operator is required to draw a box to select the desired VOI [2]. 


\section{METHODOLOGY}

The concept of computer-assisted scan planning was proposed before for human Magnetic Resonance Imaging of the heart [3][4], the brain [5], the knees [6][7] or the spine [8]. These approaches are typically based on the registration of an a-priori model of the anatomy of interest to one or a set of scout views in 3D that are acquired before the high resolution scan. Such an a-priori model, or atlas, may consist of a three dimensional structural model ([3]-[7]) or include some geometrical relationship between anatomical features [8].

The model that is used in this work is the 3D MOBY mouse atlas presented in Segars et al. 2004 [9]. It is publicly available and contains a fully segmented animal (C57BL/6). In previous work [10], we adapted this atlas for automated whole-body registration in follow-up Micro-CT scans. After articulated registration of the skeleton, anatomical bone and skin landmarks are used, together with the corresponding anatomical landmarks on the atlas, to define a Thin-PlateSpline (TPS, [11]) mapping in 3D of major organs from the atlas to the subject domain. TPS mapping was chosen since it can handle non-uniformly distributed landmarks (which anatomical landmarks usually are) and is spatially smooth. Another advantage of TPS is that it is easy to combine global and local deformations, mainly controlled by the amount and density of landmarks in a certain area. For this work, the framework has been adapted such that it is possible to perform a 3D TPS mapping of the mentioned atlas to the optical camera coordinate space, based on a set of three orthogonal 2D projections of the animal skin only (the optical camera images). Therefore, the following assumptions were made:

1) The animal contour in the optical camera side views may differ a lot for different subjects, because the animals may be put in prone or supine position into the animal holder.

2) The animal is put onto the holder in prone or supine position, with only little rotation with respect to the anteroposterior axis.

3) The lateral extent of the animal is constrained by the holder, which is visible in the top view.

4) The main focus of U-SPECT studies is on internal organs and therefore, the limbs can be disregarded.

5) The main focus of U-SPECT scan planning is on organ VOI definition and therefore small organ shape deformations that may not be anatomically realistic are not considered as critical.

Based on these assumptions, the following protocol was set up in order to obtain the anatomical landmarks that are needed for mapping the atlas organs (an overview is given in Figure 2). After the acquisition of the optical camera data as described above, some user input is required for this pilot study:

1) A coarsely drawn contour of the animal in one lateral view of the animal (white line in Figure 2),

2) The tip of the nose and the onset of the tail in the same lateral view (red crosses),

3) The left and the right boundary of the animal holder in the top view (red crosses) and

4) An indication if the animal is placed in prone or supine position.

Starting from this, a fixed number of equidistant landmarks is determined on the manually indicated lateral contour (Figure 2, A). Since the atlas is positioned in a known orientation it is possible to derive the mid-sagittal atlas crosssectional contour and sample it in the same manner (Figure 2, B). The user indicated lateral landmarks on the holder are shifted parallel to the anteroposterior axis to the middle of the animal, which is defined as half the distance between the nose and the onset of the tail, to match the location of the lateral landmarks in the atlas. Vertically, these landmarks are placed on the boundary of the holder. Subsequently the two sets of corresponding landmarks defined in the optical camera coordinate space and the atlas coordinate space respectively are used for the TPS mapping.

Based on the mapped atlas, 2D projections of the different organs are generated and displayed on top of each of the photographs (Figure 2, C). Finally, the scanner operator can select an organ of interest and subsequently a scan VOI is determined by taking the bounding box of the selected organ. To compensate for possible organ localization errors, the derived bounding box is increased in all directions before scanning, by adding some safety margin.

An example of mapped atlas organs on a subject with a bounding box increased by $50 \%$ in each direction is shown in Figure 3. 


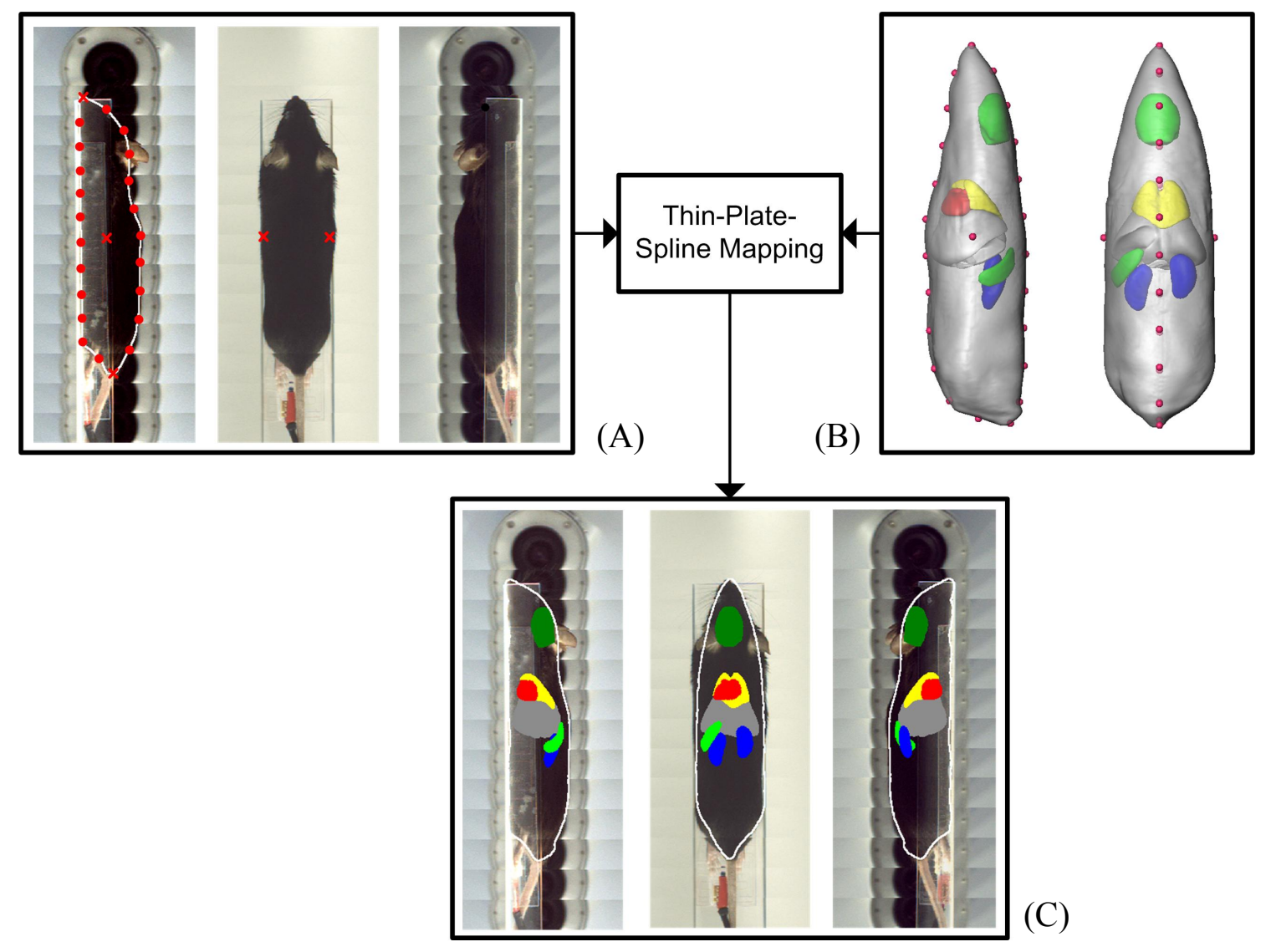

Figure 2: (A) Images taken from the left, top and right optical camera showing the user defined contour (white) and landmarks (crosses). The dots indicate the automatically determined landmarks. (B) The modified atlas and the atlas landmarks. (C) Optical camera images with an overlay of the registered atlas containing the skin, the brain, the lungs, the heart, the spleen, the liver and the kidneys. For better visibility, the part of the lung that would obstruct the heart is omitted.

\section{EXPERIMENTAL SETUP}

To investigate to what degree the atlas organs correspond to the actual organs after mapping, their true location and extent have to be known. This information cannot be obtained from the optical camera images since these only show the animal body surface and no internal structures. Using U-SPECT for this purpose would in principle be possible due to its high resolution, but this would require several different tracers for different organs and above that require a long acquisition time for highly focused whole-body acquisition. Micro-CT however offers a fast way to obtain a highresolution anatomical reference. Therefore datasets consisting of two optical camera images (left and top view) taken with the U-SPECT scanner and a micro-CT dataset (U-CT, MILabs, The Netherlands) of two different mice (C57BL/6J) in prone and supine position were acquired ex-vivo. Note that, since only two views are needed for the automated organ mapping, the right optical camera was obstructed for this pilot study to enhance the contrast between the animal and the background. The resolution of the datasets was $165 \times 165 \mu \mathrm{m}^{2}$ for the optical camera images and $83 \times 83 \times 83 \mu \mathrm{m}^{3}$ for the Micro-CT data. The latter was in addition downsampled by averaging with a factor 2, yielding a resolution of $166 \mathrm{x}$ $166 \times 166 \mu \mathrm{m}^{3}$. To ensure an identical animal posture in both modalities, the same holder was used in both scanners. The rigid transformation between the scanner coordinate spaces was derived using manually located fiducial markers that are visible in both modalities (arrows in Figure 3) and applying Procrustes alignment. Anatomical references of the brain (derived from the skull), the lungs, the heart and the kidneys were determined from the Micro-CT datasets by an expert, using Amira 3.1 (Mercury Computer Systems, Chelmsford, USA). Other organs like the liver and the spleen do not show 
sufficient contrast in Micro-CT data, without contrast enhancement, for segmentation and were therefore not considered. Subsequently, the left and top view optical camera images were used to determine the body contour landmarks (19) and the lateral landmarks (2) respectively. Together with the corresponding atlas landmark set, the transformation function for mapping the atlas to the optical camera coordinate space was derived using TPS interpolation.

For quantitative analysis of the mapping result, the Dice index s [12] was calculated in 3D. This measure takes two individual volumes $\mathrm{V}_{1}$ and $\mathrm{V}_{2}$ as well as their overlap into account and is defined as follows:

$$
s=2 \cdot \frac{V_{1} \cap V_{2}}{V_{1}+V_{2}}
$$

\section{RESULTS}

Qualitative examples of the mapped atlas organs, overlaid on the photographs, are given in Figure 3. The volumes of the organs of all four datasets as well as the calculated dice indices are reported in Table 1. To additionally evaluate to what extent the bounding box derived from a mapped organ has to be increased to entirely contain the real organ of interest, the minimum (isotropic) scaling factor of the bounding box was determined. Since the volumes of the mapped atlas and the real organs sometimes differ to a larger extent (Table 1), the minimum (isotropic) scaling factor of a bounding box based on a real organ, that encloses a mapped organ entirely, is presented as well. These results are reported in Table 2.

Table 1: Volumes of the mapped and real organs as well as the dice indices of overlap between them for two different subjects in prone and supine body posture. The minimum values for each particular organ are given in bold type.

\begin{tabular}{|c|c|c|c|c|c|c|c|c|c|c|c|c|}
\hline & \multicolumn{3}{|c|}{ Subject 1 prone } & \multicolumn{3}{|c|}{ Subject 1 supine } & \multicolumn{3}{|c|}{ Subject 2 prone } & \multicolumn{3}{|c|}{ Subject 2 supine } \\
\hline & Original & Mapped & Dice & Original & Mapped & Dice & Original & Mapped & Dice & Original & Mapped & Dice \\
\hline Brain & 351,86 & 659,49 & 0,62 & 359,16 & 665,63 & 0,53 & 407,33 & 749,24 & 0,67 & 402,77 & 657,32 & 0,67 \\
\hline Heart & 302,22 & 290,35 & 0,42 & 302,95 & 293,56 & 0,32 & 286,11 & 297,18 & 0,58 & 282,62 & 296,12 & 0,76 \\
\hline Kidneys & 303,12 & 333,43 & 0,38 & 307,90 & 297,85 & 0,53 & 403,53 & 404,72 & 0,27 & 408,90 & 334,27 & 0,53 \\
\hline Lungs & 380,80 & 520,39 & 0,54 & 398,56 & 497,39 & 0,51 & 621,27 & 526,42 & 0,54 & 596,58 & 504,73 & 0,54 \\
\hline
\end{tabular}

Table 2: Minimum isotropic scaling factors that are necessary to increase the bounding box based on a mapped/real organ to entirely enclose the real/mapped organ. The maximum scaling factor for bounding boxes based on either case are printed in bold type. Note that the given numbers indicate a scaling in all directions (positive and negative), with respect to the center of the bounding box.

\begin{tabular}{|l|l|c|c|c|c|}
\hline \multirow{2}{*}{ Brain } & $\begin{array}{l}\text { Bounding box } \\
\text { reference }\end{array}$ & $\begin{array}{c}\text { S1 } \\
\text { prone }\end{array}$ & $\begin{array}{c}\text { S1 } \\
\text { supine }\end{array}$ & $\begin{array}{c}\text { S2 } \\
\text { prone }\end{array}$ & $\begin{array}{c}\text { S2 } \\
\text { supine }\end{array}$ \\
\cline { 2 - 6 } & Mapped organ & 1,27 & 1,25 & 1,14 & 1,16 \\
\cline { 2 - 6 } & Real organ & 1,33 & 1,31 & 1,17 & 1,17 \\
\hline \multirow{2}{*}{ Keart } & Mapped organ & 1,35 & $\mathbf{1 , 4 7}$ & 1,26 & 1,16 \\
\cline { 2 - 6 } & Real organ & 1,32 & 1,43 & 1,23 & 1,17 \\
\hline \multirow{2}{*}{ Lungs } & Mapped organ & 1,39 & 1,33 & 1,23 & 1,29 \\
\cline { 2 - 6 } & Real organ & $\mathbf{1 , 4 7}$ & 1,44 & 1,25 & 1,26 \\
\cline { 2 - 6 } & Mapped organ & 1,14 & 1,16 & 1,16 & 1,25 \\
\cline { 2 - 6 } & Real organ & 1,13 & 1,14 & 1,15 & 1,22 \\
\hline
\end{tabular}




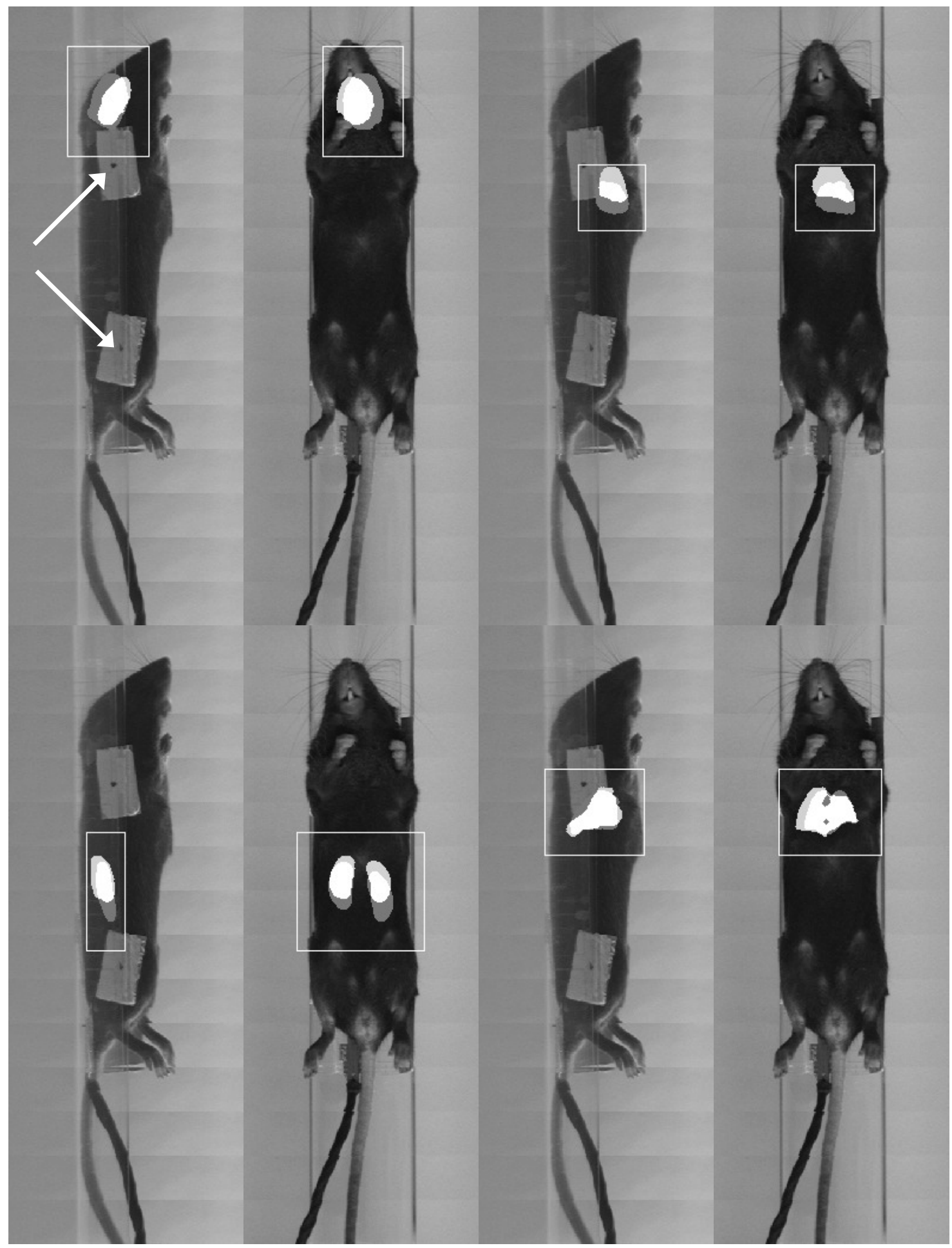

Figure 3: Organ mapping results for subject 1 in supine position. Shown are overlays of optical camera images (converted to grayscale), mapped atlas organs (dark gray), segmented organs from CT (light gray), their overlapping regions (white) and bounding boxes (derived from the mapped organ) with 50\% safety margin in each direction. The white arrows indicate the fiducial markers that were used for registration of the optical camera and the CT coordinate reference spaces. 


\section{DISCUSSION}

A qualitative analysis of the results given in Figure 3 reveals that a good mapping of the organs of interest could be obtained, without organs with unrealistic shape deformations. The quantitative results presented in Table 1 show that the mapped organ volumes of the heart and the kidneys are very similar to the real volumes. As for the brain, the mapped organ volume is systematically too large. As a result we can conclude that the brain as included in the atlas is represented relatively larger than the brains of the used subjects. The lung volumes do not show a systematic discrepancy since for subject one the mapped volumes are larger and for subject two the volumes are smaller. However the similar dice indices for either subject indicate that the location of the mapped organ is close to the real organ in both cases. The dice indices show that especially the brain and the lungs can be located properly, while the heart and the kidneys occasionally yield somewhat lower values. One thing that is remarkable for the kidney dice indices is that the ones for the prone subject seem to be worse than those for the supine subjects. As stated in [9], the atlas data was acquired in supine position as well, using a holder with similar shape than used for our experiments which may explain this difference.

The mapping results in Figure 3 reveal that the heart and the kidneys are mapped too far towards the posterior end of the animal. To investigate further on this, the relative distance of the Centers of Gravity of the real and the mapped organs was calculated for all cases. Although detailed results are not shown, we found a consistent shift of the heart $(1.2 \pm 0.7$ $\mathrm{mm})$ and the kidneys $(1.5 \pm 0.7 \mathrm{~mm})$ with respect to the anteroposterior axis such that these organs are mapped too far towards the posterior end (which is indicated by a positive shift). The amplitude of the shift suggests that it appears systematically for other datasets as well. However this has to be confirmed using a more extensive subject group. As for the brain and the lungs, the shift was smaller in this direction $(-0.01 \pm 0.3 \mathrm{~mm}$ and $-0.6 \pm 0.6 \mathrm{~mm})$ and there may exist only a marginal systematic shift. The same seems to be true for shifts with respect to the dorsoventral and the left-right axis respectively.

The results of the second experiment are presented in Table 2. It appears that all organs for all subjects can be localized using a safety margin of 50\% in each direction i.e. using a bounding box twice as big as the bounding box of the mapped organ. This increased bounding box can subsequently be used for scan planning purpose, as depicted in Figure 3 by means of frames around the organs of interest. Note that to further optimize the scanning VOI, the animal skin boundaries should be taken into account as well, which e.g. for the kidneys should lead to a significantly decreased scan VOI. To our knowledge, the size of a VOI that is determined using our method is about the size of a VOI that a scanner operator would define at least, especially for scanning the heart and the kidneys. As with the dice indices it appears that the worst results occur for the heart and the kidneys. Again, this may be due to the shift of the mapped organs with respect to the real organs. Consequently the maximum scaling factors are in most cases the ones that are needed to increase the bounding box with respect to the anteroposterior axis.

\section{CONCLUSION AND FUTURE WORK}

This work represents a pilot study towards atlas-assisted VOI definition in focused Micro-SPECT imaging. By indicating the animal contour in one lateral view and marking the holder boundaries in an orthogonal view, an animal atlas is mapped towards the target animal. Subsequently, the superposition of the mapped atlas and the optical scout images may assist the scanner operator in defining a VOI for high-resolution Micro-SPECT scanning. Since the method is not based on the SPECT data itself but on a second modality, tight VOI selection is possible even for acquisition of dynamic tracer uptake data.

A qualitative and quantitative comparison with a ground-truth Micro-CT yielded promising results because it has been shown, that adding a safety margin of $50 \%$ in each direction to a scan VOI, derived from the mapped organs, is enough to entirely encompass the real organ of interest in all cases. However, the experiments suggest that this safety margin could be decreased significantly, if systematic mapping errors would be used to adjust the animal atlas organ locations and size.

Since most of the time for SPECT scan planning using the presented method is needed to manually determine the contour of the animal in the side view, this step should be automated. In the current scanner setup this is complicated by the fact, that the two lateral (black) optical cameras record each other in the background, which leads to little contrast between the subject and the background, especially for animals with black fur. Therefore, the geometry of future scanners, the demand for a third optical camera on the scanner as well as the chosen animal strain will have an influence on the choice of the appropriate skin segmentation method. Ideally, one of the two optical cameras could be removed or permanently obscured. In such a case an active contours based method (e.g. [13]) should be able to segment the animal 
robustly and fast. Besides the contour detection, also the definition of the nose and tail landmark should be automated. Assuming that the animal is always positioned facing the scanner, e.g. due to a pipe delivering the anesthetic, the determination of the nose and the tail would not require manual indication any more. The same holds for the lateral holder landmarks if the system is fully calibrated and if one can assume that the location of the holder boundaries on the optical camera images only differs marginally over time.

This pilot study was performed on animals ex-vivo, but should ultimately be used for functional imaging i.e. in-vivo. Therefore, in the future we will focus on repeating the demonstrated experiments on living animals as well. In addition to the Micro-CT data for each subject we also plan to acquire Micro-SPECT data for specific organs, based on the automated VOI selection, and compare it to datasets acquired with expert-annotated VOIs. Since the aim of the atlas mapping is scan planning of particular organs, rather than obtaining a dense and consistent mapping of the entire animal body, the location of organs, for which a systematic dislocation was detected during the mapping, could be adjusted accordingly in the atlas.

\section{REFERENCES}

[1] Vastenhouw B., "Simulation, construction and application of focused pinhole small animal SPECT", PhD Thesis, University of Utrecht, The Netherlands, ISBN-13: 978-90-393-4941-0 (2008)

[2] Van der Have F., Vastenhouw B., Ramakers R. M., Branderhorst W. J., Krah J. O., Ji C., Staelens S. G., Beekman F. J., "U-SPECT-II: An Ultra-High-Resolution Device for Molecular Small-Animal Imaging", J. Nucl.Med., in press (2009)

[3] Lelieveldt B. P. F., van der Geest R. J., Lamb H. J., Kayser H. W. M., Reiber J. H. C., "Automated, observer independent acquisition of cardiac MR short-axis views: a pilot study", Radiology, vol. 221(2), pp 537-542 (2001)

[4] Lelieveldt B. P. F., Sonka M., Bolinger L., Scholz T. D., Kayser H. W. M., van der Geest R. J., Reiber J. H. C., "Anatomical Modeling with Fuzzy Implicit Surface Templates: Application to Automated Localization of the Heart and Lungs in Thoracic MR Volumes", Computer Vision and Image Understanding, 80, pp 1-20 (2000)

[5] Itti L., Chang L., Ernst T., "Automatic scan prescription for brain MRI", Magnetic Resonance in Medicine, 45(3), pp 486-494 (2001)

[6] Bystrov D., PekarV., Young S., Dries S. P. M., Heese H. S., van Muiswinkel A. M., "Automated planning of MRI scans of knee joints", Proc. SPIE Medical Imaging, San Diego, pp 61441-M-1 - 61441-M-8 (2007)

[7] Lecouvet F. E., Claus J., Schmitz P., Denolin V., Bos C., Berg B. C. V., "Clinical evaluation of automated scan prescription of knee MR images", J Magn Reson Imaging, 29, pp 141-145 (2009)

[8] Pekar V., Bystrov D., Heese H. S., Dries S. P. M., Schmidt S., Grewer R., den Harder C. J., Bergmans R. C., Simonetti A. W., van Muiswinkel A. M., "Automated Planning of Scan Geometries in Spine MRI Scans", proc. MICCAI, LNCS 4791, pp60-608 (2007)

[9] Segars W. P., Tsui B. M. W., Frey E. C., Johnson G. A., Berr S. S., "Development of a 4D digital mouse phantom for molecular imaging research", Molecular Imaging and Biology, 6(3), pp 149-159 (2004)

[10] Baiker M., Dijkstra J., Que I., Löwik C. W. G. M., Reiber J. H. C., Lelieveldt B. P. F., "Organ approximation in $\mu \mathrm{CT}$ data with low soft tissue contrast using an articulated whole-body atlas", Proc. IEEE Intl. Symp. on Biomedical Imaging, pp 1267-1270 (2008)

[11] Bookstein F., "Principal Warps: Thin-Plate Splines and the Decomposition of Deformations", IEEE Transactions on Pattern Analysis and Machine Intelligence, 11, pp 567-585 (1989)

[12] Zijdenbos A. P., Dawant B. M., Margolin R. A., "Morphometric analysis of white-matter lesions in MR-images Method and validation", IEEE Transactions on Medical Imaging, 13(4), pp 716-724 (1994)

[13] Xu C., Prince J. L., "Snakes, shapes, and gradient vector flow", IEEE Transactions on Image Processing, 7(3), pp 359-369 (1998) 